\title{
Role of the Histone Acetyltransferase Rtt109 in Development and Pathogenicity of the Rice Blast Fungus
}

\author{
Seomun Kwon, ${ }^{1}$ Jaejoon Lee, ${ }^{2}$ Jongbum Jeon, ${ }^{1}$ Seongbeom Kim, ${ }^{1}$ Sook-Young Park, ${ }^{3}$ Junhyun Jeon, ${ }^{2,+}$ \\ and Yong-Hwan Lee ${ }^{1,+}$ \\ ${ }^{1}$ Department of Agricultural Biotechnology, College of Agriculture and Life Sciences, Seoul National University, Seoul 08826, \\ Korea; ${ }^{2}$ Department of Biotechnology, College of Life and Applied Sciences, Yeungnam University, Gyeongsan, Gyeongbuk \\ 38541, Korea; and ${ }^{3}$ Department of Plant Medicine, Sunchon National University, Suncheon 57922, Korea
}

Accepted 30 May 2018.

Acetylation of histone $\mathrm{H3}$ lysine 56 (H3K56) by the fungalspecific histone acetyltransferase Rtt109 plays important roles in maintaining genome integrity and surviving DNA damage. Here, we investigated the implications of Rtt109-mediated response to DNA damage on development and pathogenesis of the rice blast fungus Magnaporthe oryzae (anamorph: Pyricularia oryzae). The ortholog of Rtt109 in M. oryzae (MoRtt109) was found via sequence homology and its functionality was confirmed by phenotypic complementation of the Saccharomyces cerevisiae Rtt109 deletion strain. Targeted deletion of MoRtt109 resulted in a significant reduction in acetylation of $\mathrm{H3K56}$ and rendered the fungus defective in hyphal growth and asexual reproduction. Furthermore, the deletion mutant displayed hypersensitivity to genotoxic agents, confirming the conserved importance of Rtt109 in genome integrity maintenance and genotoxic stress tolerance. Elevated expression of DNA repair genes and the results of the comet assay were consistent with constitutive endogenous DNA damage. Although the conidia produced from the mutant were not impaired in germination and appressorium morphogenesis, the mutant was significantly less pathogenic on rice leaves. Transcriptomic analysis provided insight into the factors underlying phenotypic defects that are associated with deficiency of H3K56 acetylation. Overall, our results indicate that MoRtt109 is a conserved histone acetyltransferase that affects proliferation and asexual fecundity of $M$. oryzae through maintenance of genome integrity and response to DNA damage.

Rtt109 (regulator of Ty1 transposition 109) is a fungalspecific histone acetyltransferase (HAT) responsible for histone H3 lysine-56 acetylation (H3K56ac) (Scholes et al. 2001) and functions predominantly in genome integrity maintenance. For

Current address for Seomun Kwon: Institute for Microbiology, Cluster of Excellence in Plant Sciences, Heinrich-Heine University, 40205 Düsseldorf, Germany

${ }^{\dagger}$ Corresponding authors: J. Jeon; E-mail: jjeon@yu.ac.kr and Y.-H. Lee; E-mail: yonglee@snu.ac.kr

Funding: This work was supported by Basic Science Research Program through the National Research Foundation of Korea (NRF) funded by the Ministry of Education, Science and Technology (2012R1A6A3A04038022).

*The $\boldsymbol{e}$-Xtra logo stands for "electronic extra" and indicates that six supplementary figures and four supplementary tables are published online.

@ 2018 The American Phytopathological Society
DNA replication and repair, the chromatin must be disassembled for the DNA to be accessible to appropriate machineries and then reassembled upon completion (Smerdon 1991). H3K56 acetylation by Rtt109 facilitates nucleosome assembly during DNA replication and repair by enhancing the association of $\mathrm{H} 3-\mathrm{H} 4$ dimers to chromatin assembly factors for efficient deposition onto the DNA (Chen et al. 2008; Li et al. 2008). Within the nucleosome, H3K56ac mediates looser DNA-histone interaction (Neumann et al. 2009), allowing greater flexibility and accessibility to remodeling factors for reorganization of the chromatin (Downs 2008). The function of Rtt109 associated with genome and chromatin structural integrity has been extensively studied in the model yeast organisms.

More recently, in clinically important fungi, the focus has been on understanding how Rtt109 affects infection and developmental processes to combat fungal diseases (Dahlin et al. 2014; Kottom et al. 2011; Lopes da Rosa et al. 2010; Suwunnakorn et al. 2015; Wurtele et al. 2010). For example, Rtt109 was shown to be important for development and pathogenesis of Candida albicans and Penicillium marneffei. In particular, loss of Rtt109 and, consequently, H3K56ac in C. albicans resulted in hypersensitivity to DNA damaging agents and misregulation of carbohydrate metabolism genes, which in turn accounts for increased susceptibility of the mutant to the genotoxic effects of host macrophage-derived reactive oxygen species (ROS) (Dahlin et al. 2014; Lopes da Rosa et al. 2013). Importance of Rtt109 in fungal physiology and development, in combination with its exclusivity in the fungal kingdom, made it an attractive target for development of antifungal inhibitors against opportunistic human pathogens (Lopes da Rosa et al. 2010, 2013; Suwunnakorn et al. 2015).

In contrast to the human pathogenic counterparts, the role of Rtt109 in plant-pathogenic fungi has not been elucidated to date, despite the obvious potential to target Rtt109 for development of disease-resistant plants and agrochemicals. Here, we set out to study the contribution of MoRtt109 in fungal development and pathogenesis of the rice blast fungus Magnaporthe oryzae (anamorph: Pyricularia oryzae), a historically and economically important plant pathogen (Dean et al. 2012; Talbot 2003). Rice blast infection begins when disseminated asexual spores called conidia land and germinate on the leaf surface of rice plants. Once the fungus senses the presence of a potential host through recognition of environmental cues such as surface hydrophobicity, it subsequently undergoes differentiation at the germ tip to form a specialized infection structure, called the appressorium (Ryder and Talbot 2015). The fungus then mechanically breaches the cuticular layer of the host cell with a penetration peg, driven by turgor pressure generated in 
the appressorium and colonizes host tissues with ramifying, bulbous, invasive hyphae (Kankanala et al. 2007). Consequently, the infection leads to destruction of young seedlings or grain-bearing structures of adult plants (Howard and Valent 1996). During infection, plants surmount defense responses such as production of ROS. The ability of the fungus to withstand and overcome such responses are pivotal for pathogenesis (Chi et al. 2009; Guo et al. 2010, 2011; Huang et al. 2011). Although it can be hypothesized that plant-derived ROS pose stress and induce DNA damage in pathogens, the extent and nature of the damage inflicted remain unclear.

In our previous study, we had identified an ortholog of Rtt109 (MoRtt109) in M. oryzae genome by in silico analysis (Choi et al. 2015). Here, we examine the functions of MoRtt109 as a H3K56 acetyltransferase and its impact on genome integrity, development, and pathogenicity of the rice blast fungus. Furthermore, we have analyzed the transcriptome of the wild type and the deletion mutant to gain insight into the role of MoRTT109 more specific to a fungal phytopathogen, compared with the mammalian pathogens.

\section{RESULTS}

Structural and functional conservation of MoRtt109.

The Rtt109 ortholog in Magnaporthe oryzae (MGG_05969) was identified in a previous study and was designated MoRtt109. The amino-acid sequence of MoRtt109 was aligned with orthologous sequences from model fungal species and a human pathogen in which Rtt109 functionality as a H3K56 acetyltransferase had been confirmed experimentally (Supplementary Fig. S1) (Driscoll et al. 2007; Han et al. 2007; Lopes da Rosa et al. 2010; Zhang et al. 2014). The residues involved in catalysis, acetyl-
CoA binding (Tang et al. 2008), and autocatalysis (Albaugh et al. 2011; Stavropoulos et al. 2008), which have been experimentally validated in Saccharomyces cerevisiae Rtt109, were all conserved in MoRtt109. Despite 24\% overall amino acid sequence similarity between MoRtt109 and its ortholog in S. cerevisiae (ScRtt109), they were predicted to have high structural similarity with a Tm score (a metric for measuring structural similarity of two proteins) of 0.878 in I-TASSER (Yang et al. 2015) (Fig. 1A, Tm score of 1.0 indicates identical structure).

Furthermore, heterologous expression of M. oryzae Rtt109 in the yeast deletion strain (Scrtt109A) recovered the growth defect and sensitivity to hydroxyurea (HU) (Fig. 1B), a genotoxic agent and DNA replication inhibitor known to restrict the growth of yeast Scrtt109A mutant (Driscoll et al. 2007; Zhang et al. 2014). To further corroborate such functional conservation, we examined protein-to-protein interactions. In $S$. cerevisiae, interactions of Rtt109 with histone chaperones such as Asf1 and Vps75 are known to be required for it activation and functions (Keck and Pemberton 2011; Lercher et al. 2018). When we searched the M. oryzae genome, we found possible orthologs of Asf1 (68\% identity) and Vps75 (22\% identity). Although sequence identity of MoVps75 is low, presence of a nucleosome assembly protein domain (IPR002164) and low sequence identity of Rtt109 itself (24\%) strongly suggest that such interactions are highly likely to be evolutionarily conserved. When we carried out a yeast two-hybrid experiment, indeed, we were able to infer interaction between MoRtt109 and MoVps75 (Supplementary Fig. S2). However, to our surprise, interaction between MoRtt109 and MoAsf1 seemed to be much weaker and unstable despite the much higher level of sequence conservation for MoAsf1. Together, the presence of catalytically important residues, predicted structural similarity, and the
A
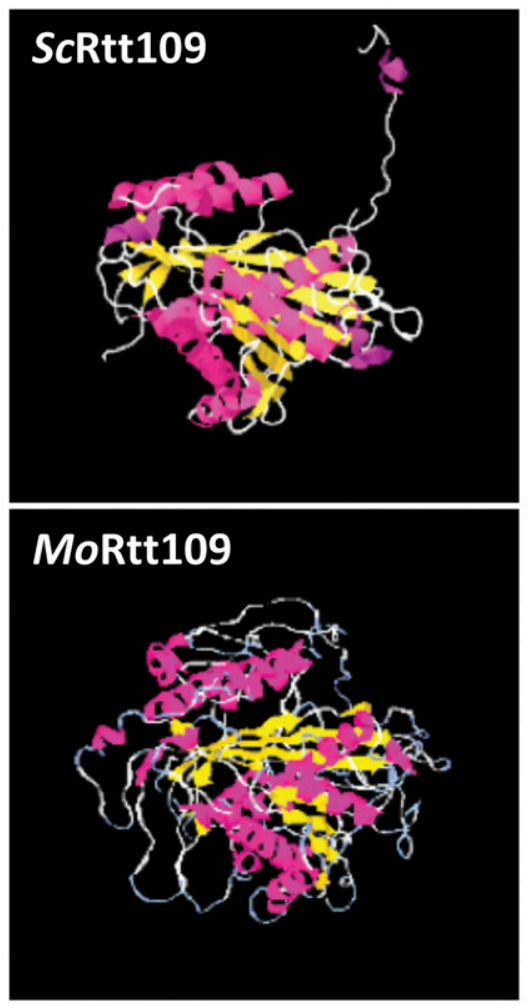

B
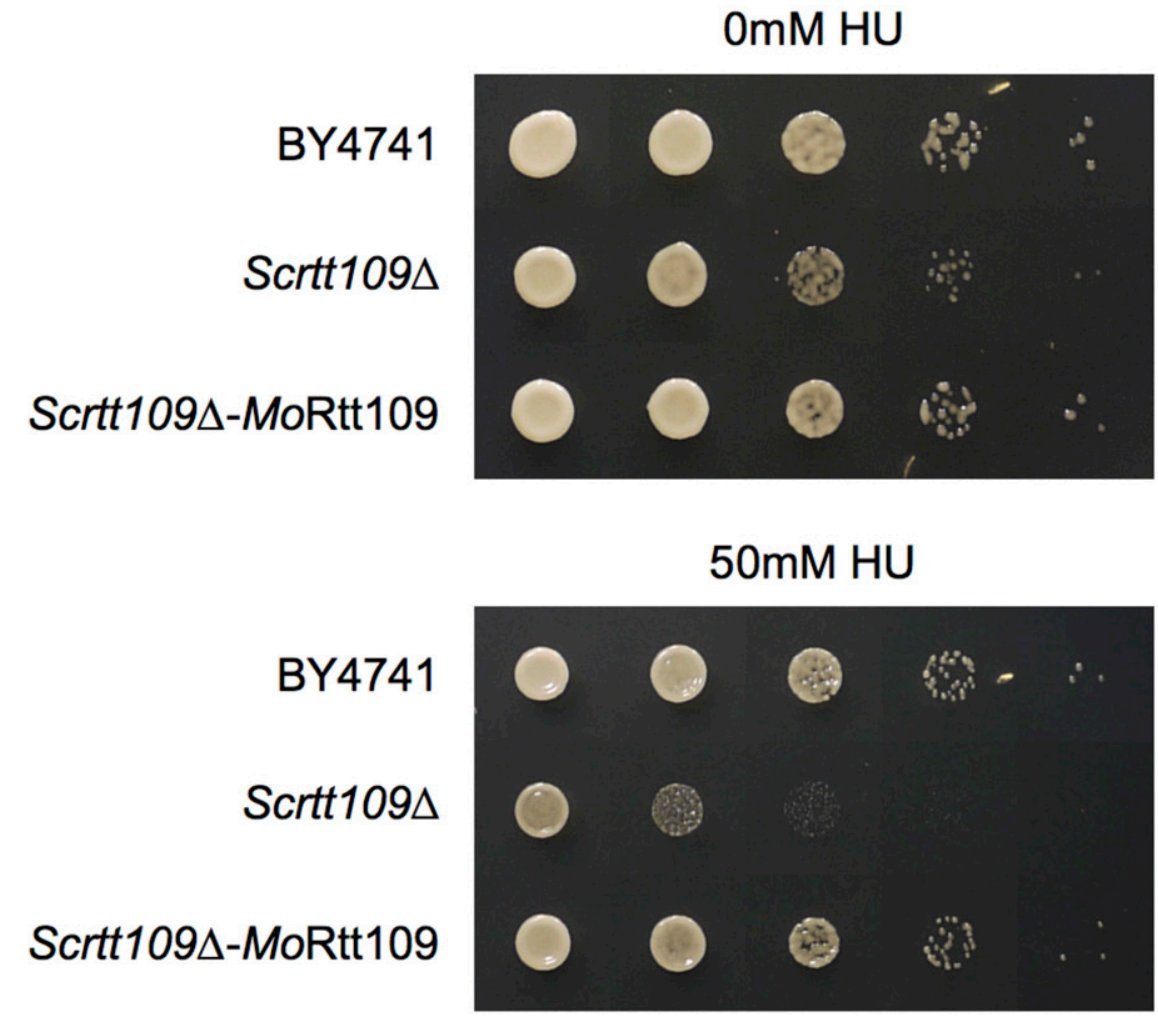

Fig. 1. Structural and functional conservation of Rtt109 between Magnaporthe oryzae and Saccharomyces cerevisiae. A, Comparison of three-dimensional structures. Structure of ScRtt109 was obtained from the Protein Data Bank and that of MoRtt109 was predicted using the I-TASSER server. B, Genetic complementation of S. cerevisiae deletion mutant (Scrtt1094) with M. oryzae Rtt109 ortholog (MoRtt109). Yeast cultures were grown to an optical density at $600 \mathrm{~nm}=0.5$, were serially diluted from 1 to $1: 10,000$, and were spotted left to right. 
genetic evidence suggest that MoRtt109 is a conserved HAT that targets H3K56.

\section{Effect of MoRtt109 deletion on hyphal growth.}

To test for Rtt109 functionality in M. oryzae, a deletion mutant (hereafter $\Delta$ Mortt109) was generated by targeted gene replacement (Fig. 2A and B). Acetylation of histone H3 lysine56 (H3K56ac) was nearly abolished in the deletion mutant and was reinstated in the genetic complementation strain (hereafter MoRtt109c) (Fig. 2C). This is in accordance with the results of Rtt109 deletion in other fungal species (Dahlin et al. 2014; Driscoll et al. 2007; Lopes da Rosa et al. 2010; Xhemalce et al. 2007; Zhang et al. 2014), attesting that MoRtt109 is, indeed, a conserved H3K56 acetyltransferase in M. oryzae.

Given its conservation across the fungal kingdom, we first examined the impact of MoRtt109 deletion on filamentous growth of the fungus. Radial growth of the $\Delta$ Mortt109 mutant was significantly reduced $(P<0.001)$ to approximately $55 \%$ of the wild type on complete medium (CM) (Fig. 3A). To ascertain the nature of the growth defect, conidia were germinated on $10 \% \mathrm{CM}$ agar and hyphal cells grown from each conidium were counted and measured. Both cell count $(P<0.001)$ and cell length $(P<0.001)$ were significantly reduced in the mutant, suggesting defects in cell division and elongation (Fig. 3B and C; Supplementary Fig. S3). The mean number of hyphal cells produced from wild-type strain KJ201 conidia was 13.3 and 13.8 for MoRtt109c, while the mutant conidia produced 8.9 cells. Mean cell length was $73.5 \mu \mathrm{m}$ for the wild type, $55.2 \mu \mathrm{m}$ for the mutant (77.5\% of the wild type), and $68.8 \mu \mathrm{m}$ for MoRtt109c. The mean lengths of whole hyphae were $974.7 \mu \mathrm{m}$ for wild type, $507.0 \mu \mathrm{m}$ for $\Delta M o r t t 109(52.0 \%$ of the wild type), and $946.92 \mu \mathrm{m}$ for MoRtt109c.
Hypersensitivity to genotoxic and ROS stresses and an elevated level of DNA damage in the absence of MoRtt109.

In $S$. cerevisiae and C. albicans, it was shown that defects in H3K56ac increased sensitivity of fungal cells to DNA damage and ROS stresses (Driscoll et al. 2007; Lopes da Rosa et al. 2010), suggesting that this modification plays an important role in DNA damage response. Therefore, we tested sensitivity of $\Delta$ Mortt 109 to the aforementioned stresses. Sensitivity was inferred from measurements of radial growth area on CM containing the stress agents as a percentage of the growth on the untreated CM (Fig. 4A). $\Delta$ Mortt109 displayed significantly greater sensitivity compared with the wild type in the presence of two genotoxins, bleomycin $(P<0.001)$ and methyl methanesulphonate (MMS) $(P<0.001)$, as well as hydrogen peroxide $\left(\mathrm{H}_{2} \mathrm{O}_{2}\right)(P<0.001)$ (Fig. 4A). Furthermore, the mutant was more sensitive than the wild type to DNA replication stresses imposed by HU $(P<0.01)$ and camptothecin $(\mathrm{CPT})(P<0.001)$, which are known to inhibit ribonucleotide reductase subunit and DNA topoisomerase I, respectively.

Since the $\Delta$ Mortt109 mutant displays hypersensitivity to DNA damage and delayed cell division (Fig. 3), it was anticipated that genome integrity is compromised in the absence of MoRtt109, so the endogenous level of nuclear DNA damage was measured by neutral comet assay (Olive and Banáth 2006). The percentage of DNA in the tail portion of the comet represents the extent of double-strand break (DSB) damage present in each protoplast cell examined. The deletion mutant protoplast population showed a significantly elevated level of DSBs $(P<0.001)$, compared with the wild-type strain (Fig. 4B), which could contribute to delayed cell division of the mutant.

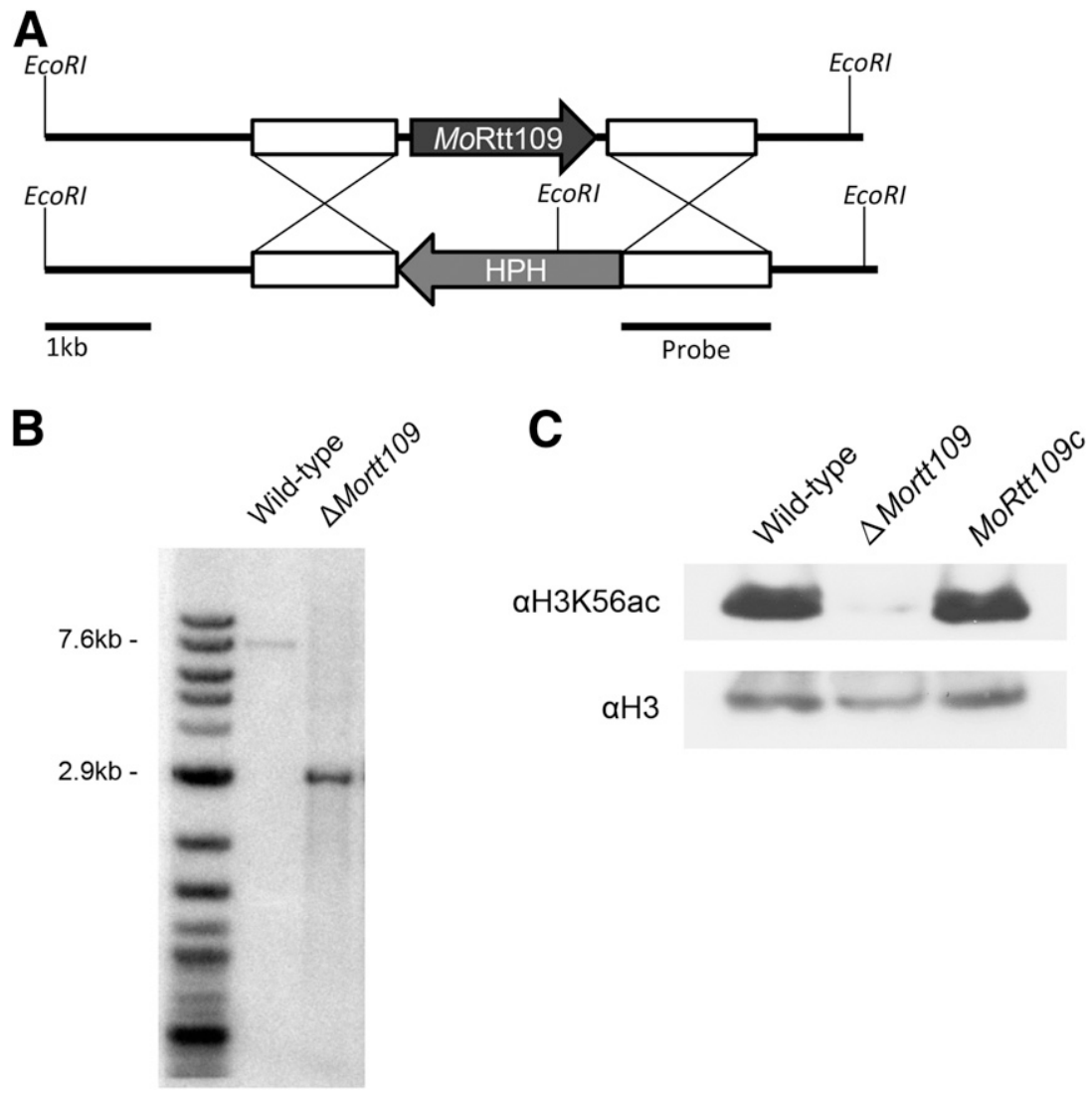

Fig. 2. Targeted deletion of MoRtt109 and its impact on the H3K56 acetylation (H3K56ac) level in Magnaporthe oryzae. A, Schematic diagram of gene replacement strategy and position of EcoRI sites. B, Southern hybridization analysis of the mutant strain using EcoRI. C, Comparison of H3K56ac level by Western blot analysis among the wild type, deletion mutant, and complementation strains. 
Asexual reproduction, germination, and appressorium formation in $\Delta$ Mortt109.

Next, we checked asexual reproduction in the mutant. Since the growth rate of mutant was much slower than the wild type, we compared asexual reproduction between wild type and the mutant by calculating conidial density, the number of spores produced per unit of colony area (square centimeters). We found that conidial density was also significantly reduced in $\Delta$ Mortt109 $(P<0.01)$ compared with the wild type (Fig. 5A). Examination of conidiophore development showed that the mutant appears to develop fewer conidiophores than the wild type (Fig. 5B). Nevertheless, mutant conidia did not show significant differences in conidial germination and appressorium formation (Supplementary Fig. S4).

\section{Requirement of MoRtt109 for full virulence in M. oryzae.}

When conidia of $\Delta$ Mortt109 were sprayed onto susceptible rice plants, the mutant was able to incite disease symptoms but produced smaller lesions than the wild type and the complementation strain, indicating that Mortt109 is required for full virulence of the fungus (Fig. 6A). Delayed hyphal growth of $\Delta$ Mortt109 prompted us to investigate the nature of the pathogenicity defect. We tested if invasive growth of the mutant could account for the reduction in lesion size by the woundinoculation method, for which spore suspension was placed directly on a wound made with a pipette tip. This should allow direct entry of the fungus into host tissues through a wound site without necessarily forming an appressorium. Despite access via wounds, the mutant still produced significantly smaller lesions $(P<0.001)$, approximately $54 \%$ the size of wild-type ones (Fig. 6B and C), which is reminiscent of the hyphal growth reduction (55\% of the wild type) on media (Fig. 3A). Correlation of the growth defect in an axenic condition with in-planta growth was further confirmed by sheath assay. Although the mutant was capable of penetrating into host cells as efficiently as the wild type, at $48 \mathrm{~h}$ postinoculation in rice sheaths, the majority (73\%) of $\Delta$ Mortt109 mutant invasive hyphae were found in the initial invaded cell, in contrast to the wild type or complementation strains, in which the hyphae were confined to the first cell in only 20 to $40 \%$ of the cases (Fig. 6D and E). It is important to note that the mutant did not seem to be compromised in the ability to suppress defense responses of host plants, since we were not able to observe accumulation of brown granules or discoloration of host cells infected with the mutant. This suggests that MoRTT109 is not directly involved in neutralizing plant defenses.

\section{Altered expression of genes involved in DNA metabolism, DNA repair, and cellular metabolism in $\Delta M o r t t 109$.}

To gain further insight into the role of MoRtt109 in $M$. oryzae, we analyzed the transcriptome (RNA-seq) of the wild type and the mutant strain grown in CM, with three biological replicates each. Our RNA-seq analysis showed that approximately $13 \%$ of the predicted gene set $(n=1,641)$ was found to be differentially expressed, with statistically significant differences and a twofold expression change cutoff. Among those differentially expressed genes (DEGs), about 55 $(n=908)$ and $45 \%(n=733)$ of DEGs were up-regulated and down-regulated in the mutant strain, respectively. DEGs include several genes involved in DNA damage response (Supplementary Table S1), almost all of which showed elevated expression in $\Delta$ Mortt 109 . In contrast, many genes related to cellular metabolism were down-regulated in the mutant. Several genes that displayed transcriptional differences in RNAseq were validated by reverse transcription-quantitative polymerase chain reaction (RT-qPCR) experiments. These genes include Rhm51, Rhm52, Rhm54, MoLig4, and MoKu80, which are involved in homologous recombination or nonhomologous end-joining DNA repair processes (Supplementary Fig. S5). Similar patterns were detected by RNA-seq and RT-qPCR (Pearson correlation coefficient $=0.87$ ), indicating that we have accurately detected gene expression changes in our experiments. Notably, we found from RNA-seq that some of the cellcycle genes are misregulated in the mutant (Supplementary Table S2). For example, MoRAD53 (MGG_01596), which is predicted to be an effector kinase for DNA replication and the DNA damage checkpoint, was down-regulated in the mutant, suggesting that reduced number and length of mycelial cells in the mutant are, at least in part, due to the faulty regulation of those genes. Given the sensitivity to $\mathrm{H}_{2} \mathrm{O}_{2}$, we also examined the expression of ROS and cell wall-related genes in the mutant. Most of the genes involved in detoxification of ROS such as $\mathrm{H}_{2} \mathrm{O}_{2}$ were not differentially expressed between wild type and the mutant (Supplementary Table S3). However, some of the cell wall-related genes showed differential expression, suggesting that hypersensitivity to $\mathrm{H}_{2} \mathrm{O}_{2}$ might be, at least in part, due to defects in the cell-wall integrity of the mutant (Supplementary Table S4). This points out that sensitivity of the
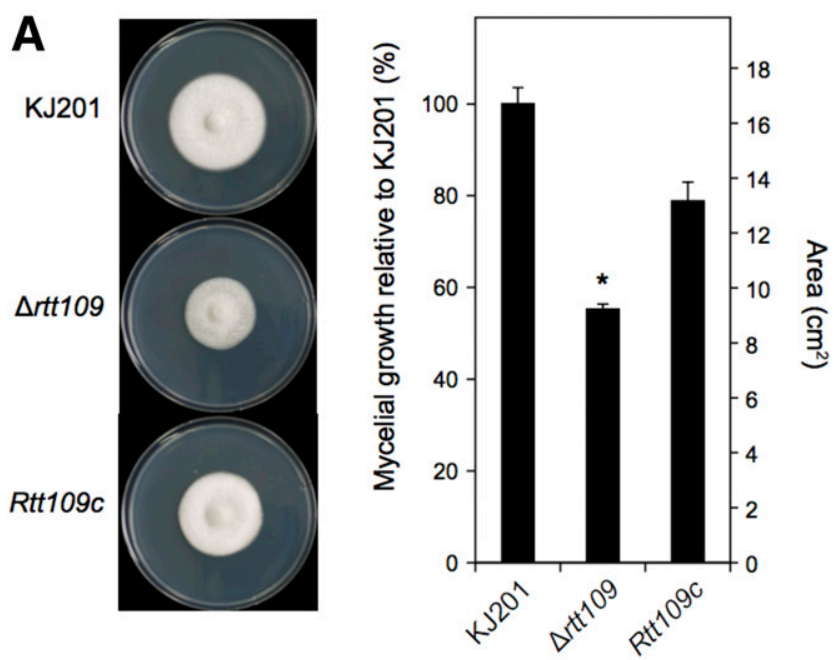

B
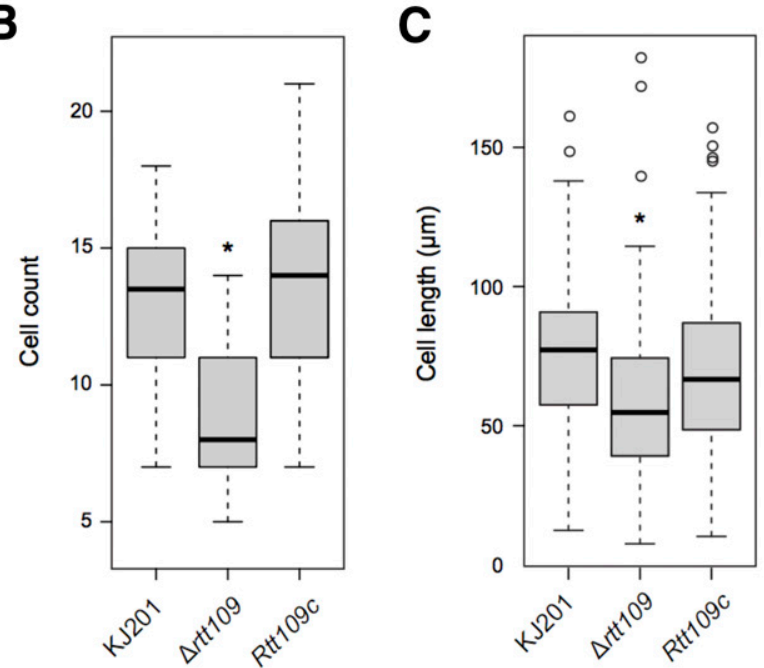

Fig. 3. Vegetative growth reduction of the $\Delta$ Mortt109 strain. A, Mycelial growth on complete agar medium on day 7. 4 Mortt 109 mycelial growth is significantly reduced to approximately $55 \%$ of the wild type. B, Cell count from hyphae grown from individual conidia at approximately $18 \mathrm{~h}$. C, Cell length measured from hyphae grown from conidia at approximately $18 \mathrm{~h}$. Cell length is measured as the distance between adjacent septa (in micrometers). Asterisk indicates statistically significant difference $(P<0.001)$. 
A

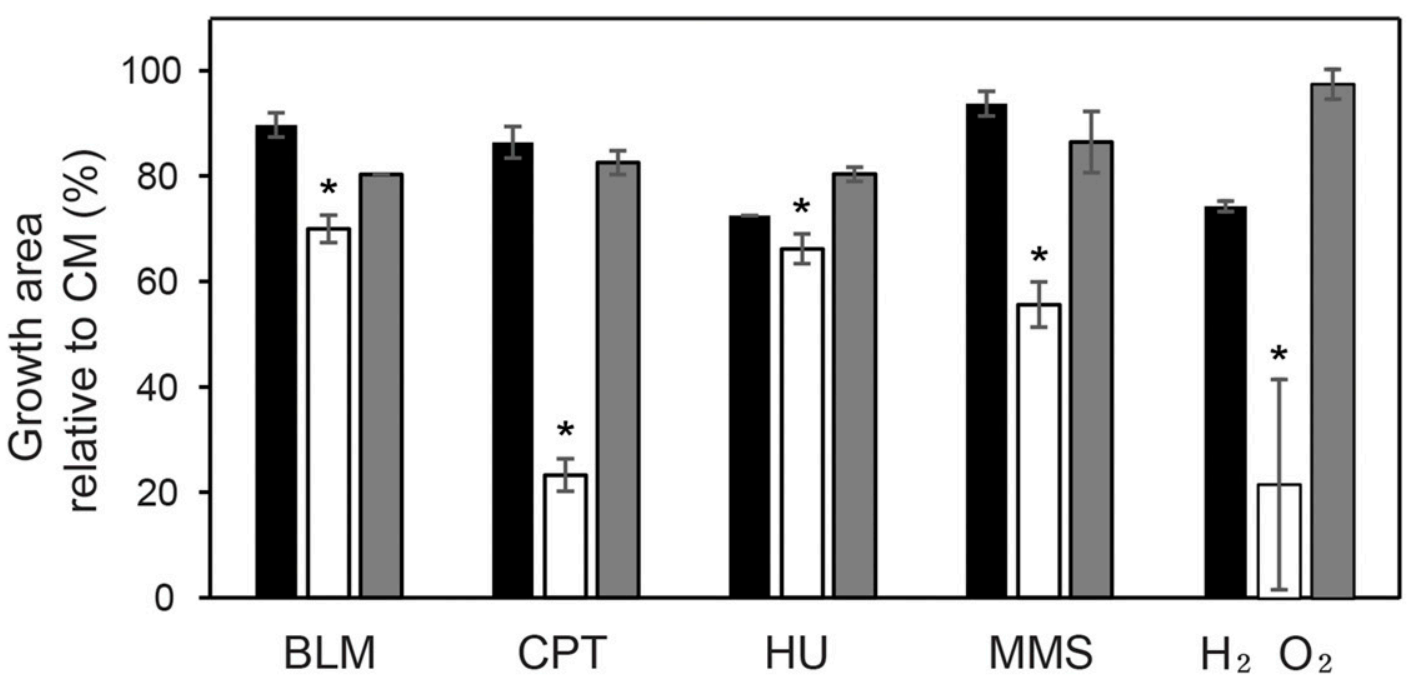

- Wild-type

口 Mortt109

MoRtt109c

B

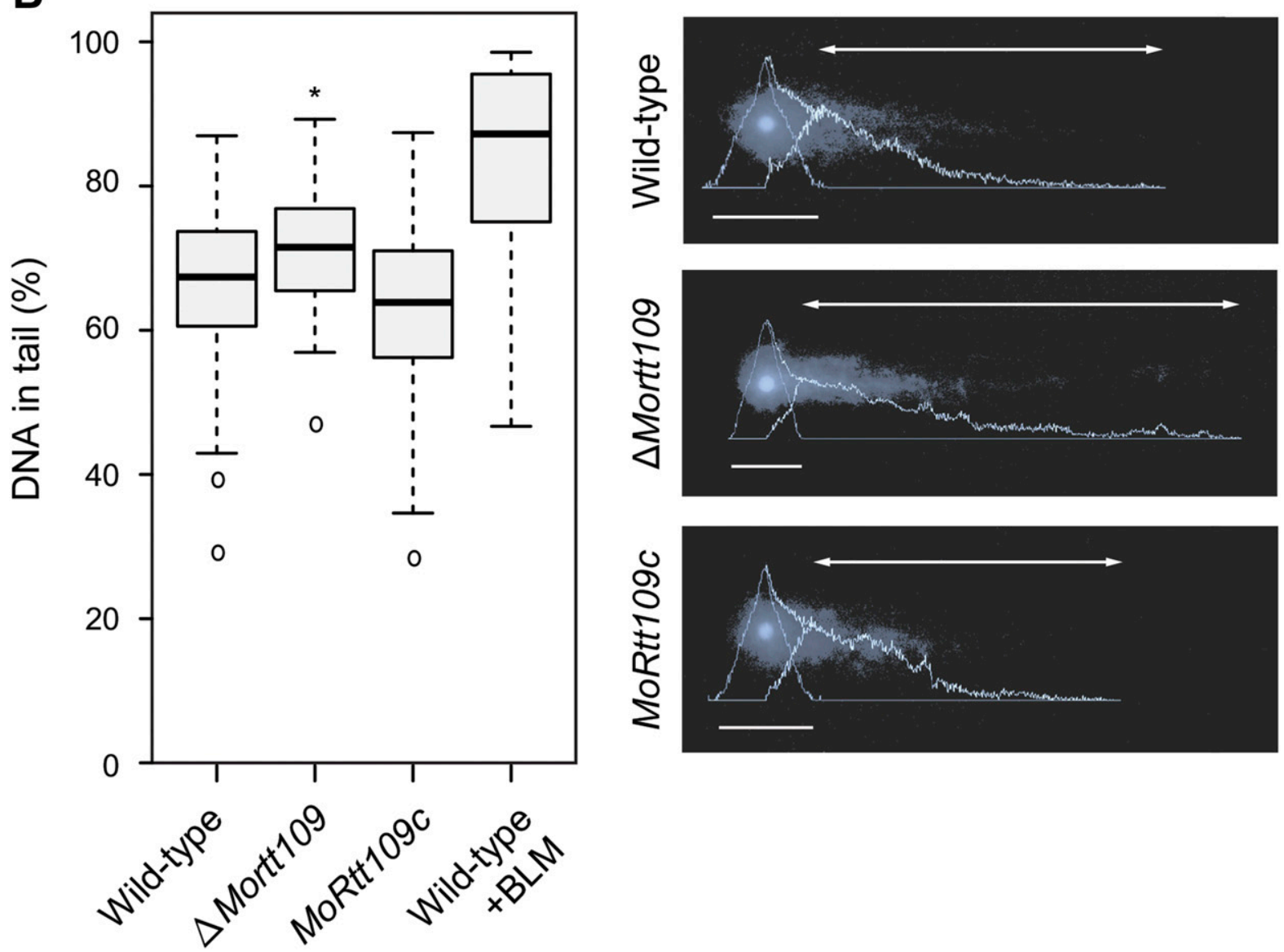

Fig. 4. Sensitivity to genotoxic and reactive oxygen species stress and endogenous DNA damage in $\triangle$ Mortt109. A, Radial growth on genotoxic stress media containing bleomycin (BLM), camptothecin (CPT), hydroxyurea (HU), methyl methanesulphonate (MMS). Radial growth area on complete medium (CM)based stress media on day 7 was measured as a percentage of growth on respective untreated CM samples. Black, white, and gray bars represent the wild type, deletion mutant, and complementation strains, respectively. B, Comparison of endogenous DNA damage levels. Neutral comet assay was carried out to detect the extent of double-strand break damage. The wild-type strain KJ201 treated with $5 \mathrm{mU}$ BLM has been used as a positive control. An asterisk indicates statistically significant difference $(P<0.001)$. 
mutant to $\mathrm{H}_{2} \mathrm{O}_{2}$ may be due to a combination of interrelated defects resulting from an imbalance of H3K56 acetylation, although it remains a possibility that the mutant cannot induce expression of ROS detoxification genes in response to the presence of $\mathrm{H}_{2} \mathrm{O}_{2}$.

To extend these observations, we carried out gene ontology (GO) term analysis to quantify the enrichment of genes associated with biological processes. Our GO term analysis showed enrichment of genes involved in cellular response to stress, DNA metabolic process, and DNA repair among up-regulated genes (Fig. 7). Unlike the upregulated genes, the downregulated genes were enriched with GO terms implicated in basic metabolism and growth, such as mycelium development, organic acid metabolism, vitamin metabolism, and most notably, amino acid metabolism (Supplementary Fig. S6). Such transcriptional output of the mutant genome suggests that the mutant strain is subjected to constitutive endogenous DNA damage and an imbalance in cellular metabolism.

\section{DISCUSSION}

In this work, we have examined for the first time the importance of the fungal-specific HAT Rtt109 in growth, development, and virulence of a plant-pathogenic fungus. First, the presence of a functional ortholog of Rtt109, responsible for $\mathrm{H} 3 \mathrm{~K} 56 \mathrm{ac}$, was confirmed in M. oryzae. It can be inferred from the correction of $S$. cerevisiae mutant phenotypes by heterologous expression of MoRtt109 (Fig. 1B), that the M. oryzae ortholog is capable of interacting with yeast histone chaperones Asf1 and Vps75, which are essential for Rtt109 functionality as a HAT (Fillingham et al. 2008). Rtt109 interactions pertinent to chromatin dynamics during DNA replication (Han et al. 2007; Li et al. 2008) and repair processes (Chen et al. 2008; Masumoto et al. 2005) discovered in yeast are likely to be conserved in the rice blast fungus as well, given the parallel phenotypic defects in growth (Fig. 3), genome integrity (Fig. 4), and genotoxin sensitivity (Fig. 4) between the M. oryzae and $S$. cerevisiae $\Delta r t t 109$ mutants (Han et al. 2007; Li et al. 2008).

Compromised genome integrity in the absence of MoRtt109 was originally expected to affect three major aspects of $M$. oryzae development, namely, hyphal growth, conidiation, and appressorium formation. Delayed cell-cycle progression was evident from the reduced number of hyphal cells produced by the M. oryzae $\Delta$ Mortt109 strain (Fig. 3). The mutant also displayed reduced conidiation, presumably due to slower growth of aerial hyphae (Fig. 5), although the conidia produced were fully functional and morphologically indistinguishable from the wild type (Fig. 5). The growth defect is supported by the findings in S. cerevisiae, that Rtt109 plays an important role in replication-fork stability and DNA-damage tolerance, especially during the $S$ phase of the cell cycle (Driscoll et al. 2007; Han et al. 2007). An increased level of spontaneous DSBs (Fig. 4B) and stalled DNA replication are expected to contribute to cell-cycle delay of the $\Delta r t t 109$ strain.

To initiate infection, $M$. oryzae conidia germinate and form a specialized structure called an appressorium, in which high turgor pressure is generated to force the penetration peg through the plant cell wall (Howard and Valent 1996; Wilson and Talbot 2009). Entry into the $S$ phase and mitosis are each known to be essential for the developmental initiation and maturation of the appressorium (Saunders et al. 2010). DNA damage can lead to cell-cycle delay before and during the $\mathrm{S}$ phase as well as before mitosis (O'Connell et al. 2000). Therefore, it was hypothesized that, if the $\Delta$ Mortt109 mutant suffers more replication stress and spontaneous DNA damage, more germlings would be delayed in appressorium initiation and maturation. However, delays in conidial germination, appressorium initiation, and maturation could not be observed for the $\Delta$ Mortt109 strain at the timepoints tested. Therefore, we conjecture that, although the degree of DNA damage suffered by the $\Delta$ Mortt109 strain affects hyphal growth (Fig. 3) and conidiation (Fig. 5), it is insufficient to delay pre-penetration development. Alternatively, there might be a mechanism that
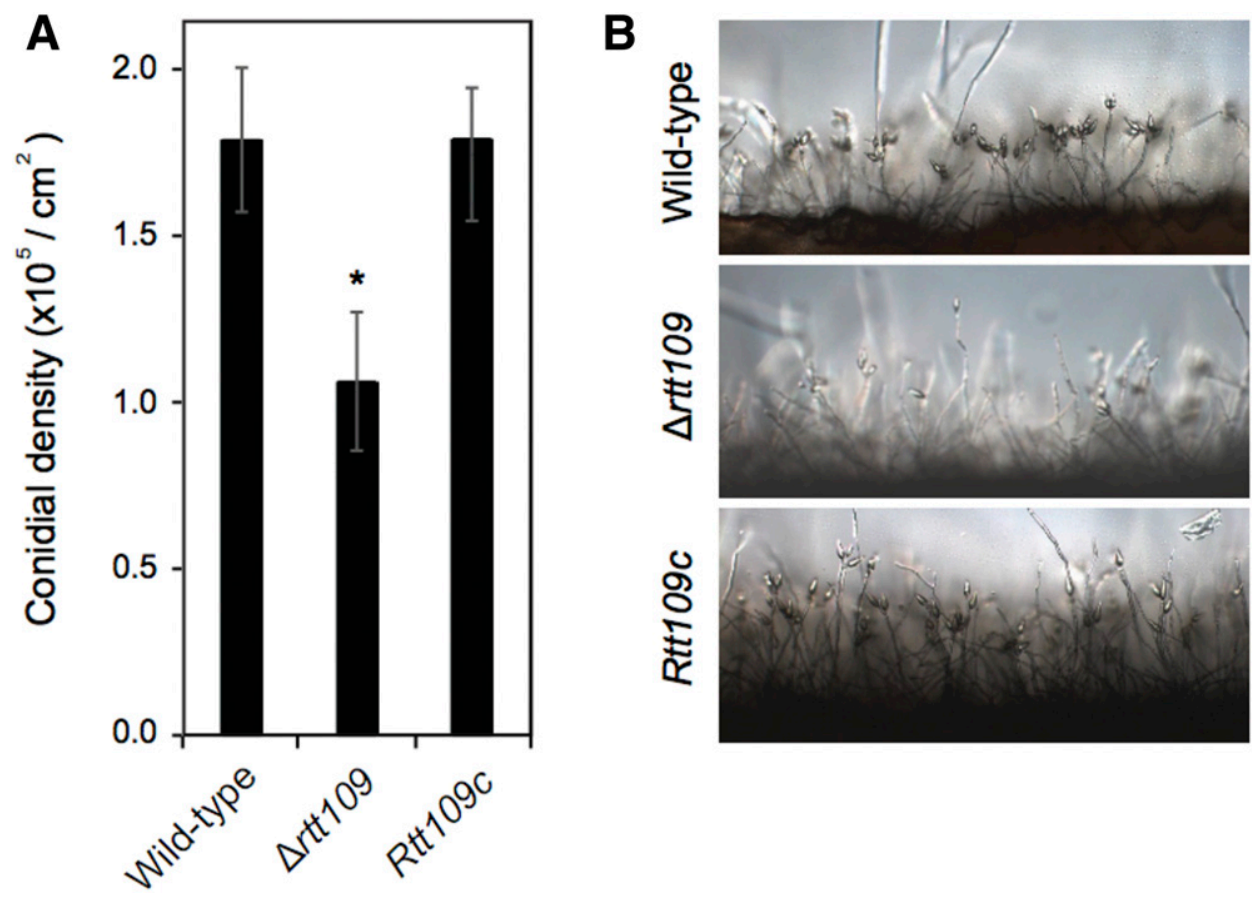

Fig. 5. Asexual reproduction in $\Delta$ Mortt109. A, Conidial density estimates from 7-day-old colonies on V8 agar medium. Conidiation is significantly reduced in $\Delta$ Mortt 109. An asterisk indicates statistically significant difference $(P<0.01)$. B, Comparison of conidiophore development and conidiation on oatmeal agar medium at $27 \mathrm{~h}$ postincubation. 
allows the fungus to bypass the DNA damage checkpoint during germination and appressorium formation.

Rtt109 is a conserved genome integrity maintenance factor implicated with DSB repair (Chen et al. 2008; Jessulat et al. 2008; Muñoz-Galván et al. 2013). Naturally, the rice blast fungus suffers DBSs throughout its life cycle (Ndindeng et al. 2010). DSB repair pathways consist of homologous recombination and nonhomologous end joining. Homologous

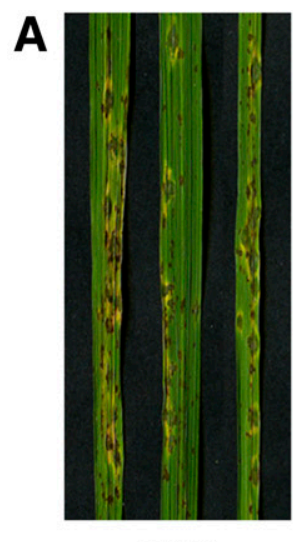

KJ201

B

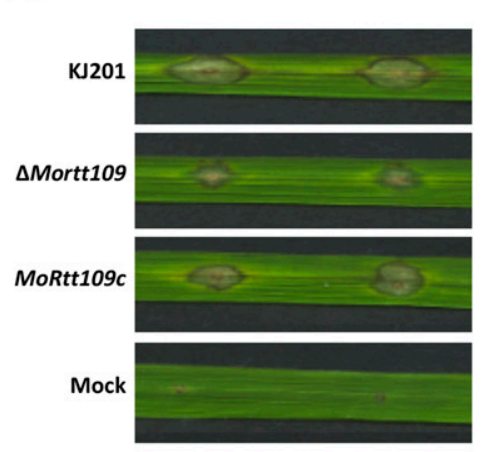

D

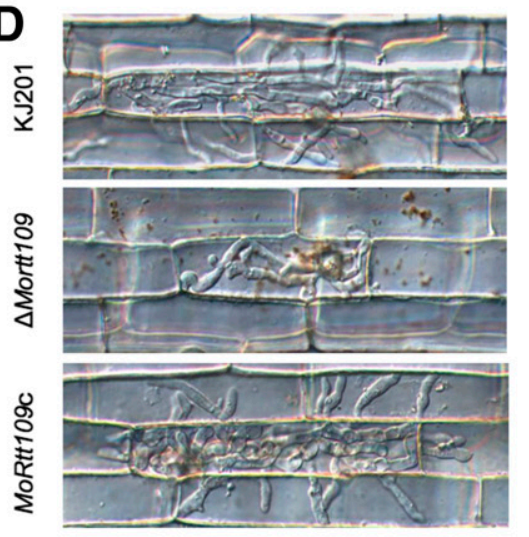

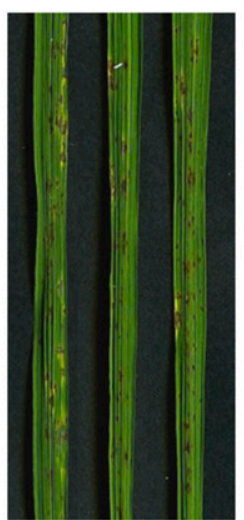

$\Delta$ Mortt109

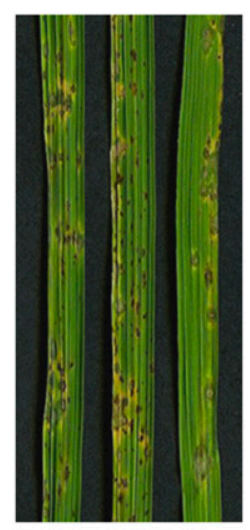

Mortt109c

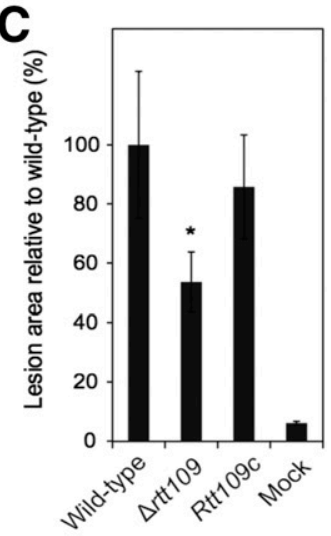

E

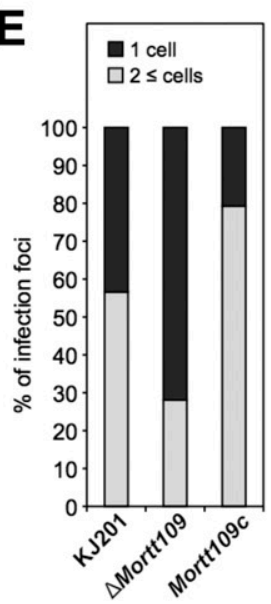

Fig. 6. Dissection of a pathogenicity defect of MoRtt109 deletion mutant. A, Lesion development in rice leaves 7 days post-spray inoculation. B, Lesion development in wound-inoculated rice leaves 5 days postinoculation. Distilled water was used for the mock experiment. C, Lesion area measured from wound-inoculated leaves shown in B. Mean area was calculated from at least four individual lesions. Asterisk indicates statistically significant difference $(P<0.001)$. D, Observation of invasive growth using rice sheath assay. Invasive growth in rice sheath cells was monitored under a microscope at $48 \mathrm{~h}$ postinoculation (hpi). E, Quantitative measure of invasive growth in rice sheath cells. Number of rice cells that the fungus has invaded at 48 hpi was counted for each strain. recombination mutants lacking Rhm51, Rhm52, and Rhm54 (RAD homolog in Magnaporthe) are severely affected in growth, infectious development, and pathogenicity (Ndindeng et al. 2010). Deletion of MoRtt109, however, did not give rise to developmental and pathogenicity defects as severe as those displayed by the homologous recombination pathway mutants. This may be explained by the function of MoRtt109 in postrepair chromatin reassembly rather than being directly involved in the DSB repair process itself. Furthermore, the general increase in expression of DNA damage repair genes in the $\Delta$ Mortt109 mutant, including ones in the DSB repair pathways, may have compensated for the liability to suffer DNA damage and inefficient repair, resulting in milder phenotypic defects.

The $\Delta$ Mortt109 mutant showed significantly reduced pathogenicity, producing lesions that measure approximately $54 \%$ of the wild-type lesions. This was in accordance with the $\Delta$ Mortt109 mutants in animal pathogens C. albicans and Penicillium marneffei, in which virulence was significantly diminished but not abolished(Lopes da Rosa et al. 2010; Suwunnakorn et al. 2015). Reduced invasive hyphal growth in the absence of pre-penetration defects suggests that the reduced growth rate is largely responsible for the attenuated pathogenicity of $\Delta$ Mortt109 and that MoRtt109-mediated processes are not responsible for coping with host-derived ROS. However, we cannot completely rule out the possibility that the mutant is rendered more sensitive to unknown stresses in planta.

It is interesting to note that our RNA-seq data not only support conserved phenotypic defects associated with Rtt109, such as endogenous DNA damage and sensitivity to genotoxins, but also reveal differences between yeast and filamentous fungi. Transcriptome analysis of $\Delta M$ ortt 109 showed that amino acid metabolisms were altered in the mutant, in contrast to the $C$. albicans mutants, in which deletion of Rtt109 resulted in misregulation of carbohydrate metabolisms. Moreover, in yeast, it was shown that deletion of Rtt109 or DNA damage and ROS can promote filamentous growth in a RAD53-dependent manner (Jiang and Kang 2003; Shi et al. 2007). In contrast, our work showed that deletion of MoRtt109 suppressed cell elongation in the filamentous fungi $M$. oryzae. Together with our RNA-seq data, this suggests that at least part of the genetic network implicating Rtt109 should be wired distinctively between yeast and filamentous fungi.

Considering the pathogenicity test results and the fact that Rtt109 is a fungal-specific enzyme, it is tempting to think that Rtt109 would serve as a target for host-induced gene silencing (HIGS) or a fungicide, although it would certainly not confer full resistance. Nonetheless, given that rice blast is a polycyclic disease (Skamnioti and Gurr 2009) and perturbation of MoRtt109 function reduces production of conidia that act as the main inoculum, it could limit the production of secondary inoculum on the infected plant, reducing the likelihood of an epidemic. Another possibility is to combine inactivation of Rtt109 with a fungicide that has a mechanism of action involving lethal oxidative stress. ROS can cause cellular damage in multiple ways including DNA DSBs and, in turn, DNA damage can raise intracellular ROS levels (Salmon et al. 2004). It is probable that the hypersensitivity of the $\triangle M$ ortt109 strain to ROS is in part due to compromised ability to repair DNA damage. In the opportunistic pathogen $C$. albicans, hypersensitivity of $\Delta r t t 109$ to ROS and genotoxic stresses has been associated with susceptibility to certain fungicidal drugs (Lopes da Rosa et al. 2010; Wurtele et al. 2010). Moreover, in yeast fungi, generation of toxic levels of intracellular ROS has been highlighted as a common pathway triggered by different classes of fungicides (Belenky et al. 2013). Also, in the plant-pathogenic fungus Mycosphaerella graminicola, transcriptional responses to azole fungicide treatment included upregulation of transcripts encoding mitochondrial respiratory 
chain components (Cools et al. 2007), which further supports oxidative stress as a convergent mechanism of fungicide-induced cell death. These lines of evidence suggest the possibility to reduce the usage of fungicides in combination with HIGS of Rtt109.

In summary, our data indicate that MoRtt109 is a conserved epigenetic component responsible for acetylation of $\mathrm{H} 3 \mathrm{~K} 56$, which, in turn, is important for growth and survival of plant-pathogenic filamentous fungi. Considering the importance of H3K56acmediated processes in DNA replication and DNA damage responses, MoRtt109 has profound implications for development and pathogenicity of the fungus. Transcriptome data provide insight into commonalities and differences in genetic context of a shared epigenetic factor between human and plant pathogenic fungi. Further elucidation of genes and mechanisms underlying MoRtt109-mediated processes would shed light on the development of novel control strategies for fungal diseases of plants.

\section{MATERIALS AND METHODS}

Magnaporthe oryzae strains and growth conditions.

KJ201 is the wild-type Magnaporthe oryzae strain used in this study, and all recombinant strains produced have the KJ201 background. $M$. oryzae strains were maintained as dried oatmeal agar stocks stored at $-75^{\circ} \mathrm{C}$ and were grown at $25^{\circ} \mathrm{C}$ under constant light on solid V8 medium (8\% V8 juice [vol/vol], $1.5 \%$ agar powder [wt/vol], pH 6.7), unless otherwise stated. Conidia used in pathogenicity tests and for observing pre-penetration development were harvested from 7-day-old cultures on solid V8 medium. Rice plants (Oryza sativa cv. Nakdong) were grown at $25^{\circ} \mathrm{C}$ under 16-h light and 8-h dark cycles. Protoplasts, DNA, RNA, and protein were all extracted from 3-day-old mycelial cultures in $\mathrm{CM}$ broth $(0.6 \%$ yeast extract, $0.6 \%$ tryptone, $1 \%$ sucrose [all wt/vol]) and were grown at $25^{\circ} \mathrm{C}$ with shaking at $150 \mathrm{rpm}$ in the dark. Conidia from 7-day-old colonies on V8A medium were used as the starting inoculum for liquid cultures.

Targeted deletion of Rtt109 and genetic complementation.

Deletion construct was designed for targeted replacement of MoRtt109 (MGG_05969) open reading frame (ORF) with hygromycin B phosphotransferase gene $(H P H)$ cassette by homologous recombination. The1.361-kb region flanking the 5' end (forward primer: GTCCTCCGCAGTGATGGGTATT; reverse primer: GCACAGGTACACTTGTTTAGAGAAGTTA TATGCGAACTCATGCCATTG) and the 1.399-kb sequence flanking the $3^{\prime}$ end of the ORF (forward primer: CCTTCAA TATCATCTTCTGTCGAGATTGCGACTTGGCTATGTTAA

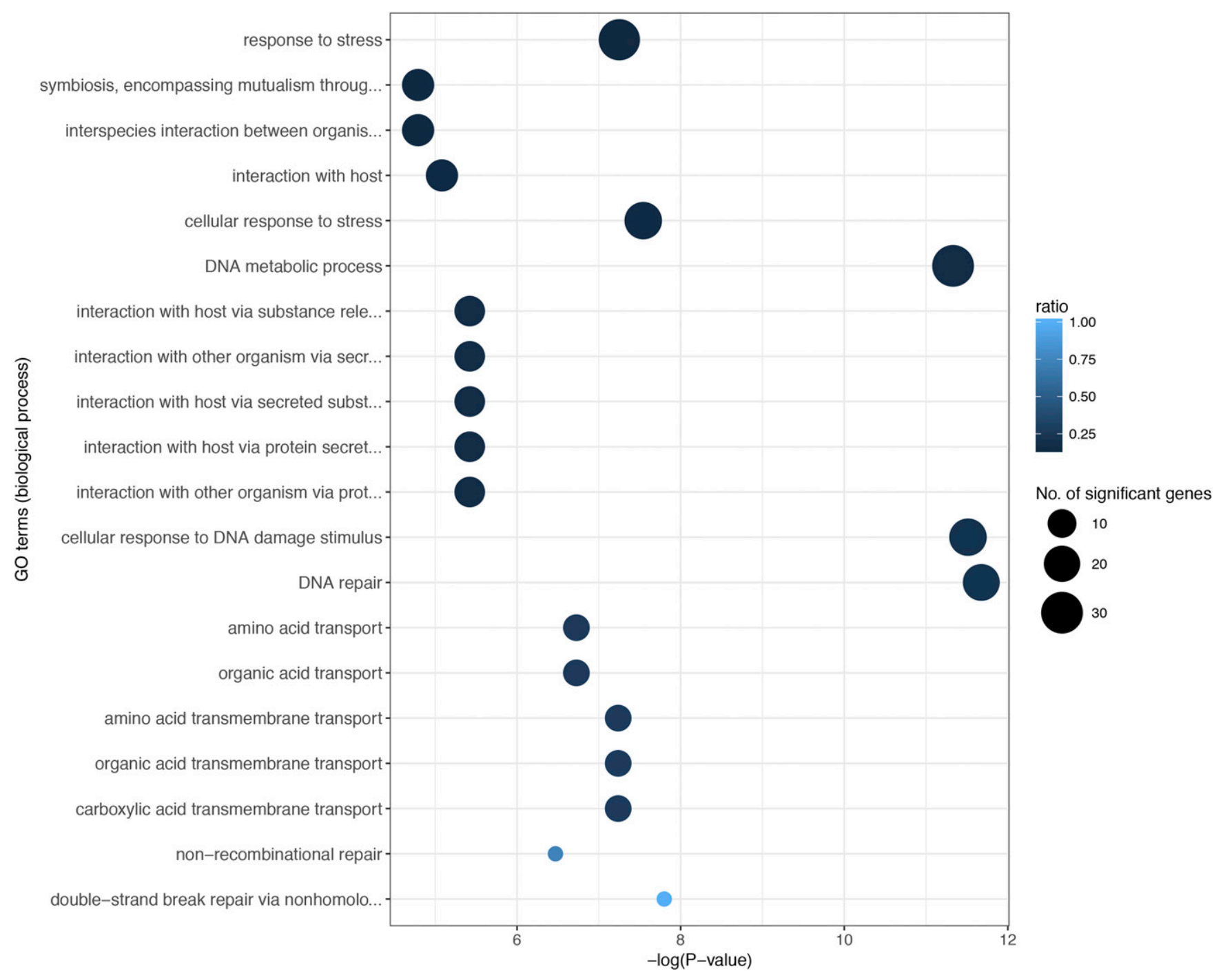

Fig. 7. Summary of gene ontology (GO) enrichment analysis (biological process). GO terms that are enriched with upregulated genes are shown (Fisher's exact test, $P<0.01$ ). The area of a circle represents the number of genes assigned to the particular GO term. Color of the circle indicates the proportion of genes assigned to the GO term in our dataset among the total number of genes having that GO term in the genome. 
CAA; reverse primer: CTAAATGGTCGCTTATCGAGTGCT) were amplified and were fused to the $H P H$ sequence by fusion PCR. The construct was introduced to the wild-type KJ201 protoplasts by polyethylene glycol-mediated transformation. Transformants were selected on solid TB3 media $(0.3 \%$ yeast extract, $0.3 \%$ casamino acid, $1 \%$ glucose, $20 \%$ sucrose, and $0.8 \%$ agar powder [all wt/vol]) containing $200 \mathrm{ppm}$ of hygromycin B and were screened by PCR, then, Southern blot to identify successful deletion mutants with a single integration event. Genetic complementation was carried out by reintroducing the MoRtt109 ORF with the aforementioned flanking regions into $\Delta$ Mortt109 mutant protoplasts together with plasmid pII99 containing the geneticin resistance marker. Complementation strains $($ MoRtt109c) were screened first on solid TB3 medium supplemented with $800 \mathrm{ppm}$ of geneticin and, then, by PCR. All strains were deposited at the Center for Fungal Genetic Resources at Seoul National University, Korea.

\section{Western blot analysis of $\mathrm{H3K56}$ acetylation.}

Total protein extracted from mycelia was separated by $12 \%$ sodium dodecyl sulfate-polyacrylamide gel electrophoresis. Blots were probed with histone $\mathrm{H} 3$ acetyl-lysine K56 antibody $(1: 2,000)$ (Active Motif) and H3 antibody as loading control (1: 2,000) (Active Motif). Pierce Fast Western blot kit, ECL substrate (Pierce Biotechnology) was used and signals were detected on X-ray films.

\section{Phenotypic complementation \\ of Saccharomyces cerevisiae $\mathrm{rtt109 \Delta}$ mutant.}

Magnaporthe oryzae MoRtt109 cDNA was amplified and cloned into pYES2 (Life Technologies), was sequenced and introduced to the Saccharomyces cerevisiae rtt109A strain Y01490 (Euroscarf). Transformants were selected on Sc-Ura medium and were confirmed by PCR. Yeast strains were grown to an optical density at $600 \mathrm{~nm}=0.5$, then, serially diluted tenfold up to 1:10,000 for spot assay. Three microliters of each of the dilution series was spotted onto inductive solid Sc minimal medium $(6.7 \%$ yeast nitrogen base without amino acids, $2 \%$ drop-out mix, $2 \%$ galactose, and $1 \%$ raffinose), with or without $50 \mathrm{mM} \mathrm{HU}$, then incubated at $30^{\circ} \mathrm{C}$ for 3 days.

\section{Yeast two-hybrid experiment.}

The ProQuest two-hybrid system (Invitrogen) was used to identify interaction between proteins in this study (MoRtt109MoVps75 or MoRtt109-MoAsf1). The experiments used in this study were carried out according to the standard protocol for this system. cDNAs encoding bait and prey proteins were amplified and were cloned using the following primers: Arf1 forward: GGGGACAAGTTTGTACAAAAAAGCAGGCTCT ATGTCTGTCGTAACGCTACT, Arf1 reverse: GGGGACCAC TTTGTACAAGAAAGCTGGTCTTAGTGAGACATAATCTC GGC; Rtt109 forward: GGGGACAAGTTTGTACAAAAAAG CAGGCTCTATGACAACAACTATGGCTACC, Rtt109 reverse: GGGGACCACTTTGTACAAGAAAGCTGGTCTCATTGTCC TTGTTCTTTTTTTC; Vps75 forward: GGGGACAAGTTTGT ACAAAAAAGCAGGCTCTATGTCTTCGACATCGACCCC, Vps75 reverse: GGGGACCACTTTGTACAAGAAAGCTGGT CTTACTTCTTCTGCTTTTTGGCTG. The bait plasmid pDEST32Rtt109 and the prey plasmids pDEST22-Asf1 or pDEST22-VPS75 were transformed into S. cerevisiae Mav203 cells. Empty pDEST32 plasmid and prey plasmids pDEST22-Asf1 or pDEST22-VPS75 were transformed into S. cerevisiae Mav203 cells to detect self-activation of the prey vector. The transformants were screened on Sc-Leu-Trp plates. Cells were then transferred onto sterile nylon membrane, which were plated on yeast extract-peptone-dextrose plus adenine medium to determine $\beta$-galactosidase activities using an X-gal assay.
Growth test, hyphal cell counting, and cell-length measurements.

Plugs ( $4 \mathrm{~mm}$ in diameter) taken from the periphery of colonies grown on solid minimal medium (MM) (1\% glucose, $0.6 \% \mathrm{NaNO}_{3}, 0.05 \% \mathrm{KCl}, 0.05 \% \mathrm{MgSO}_{4}, 0.15 \% \mathrm{KH}_{2} \mathrm{PO}_{4}$, $0.1 \%$ trace element [vol/vol], $0.1 \%$ vitamin supplement [vol/vol], pH 6.5 [all wt/vol, unless otherwise stated]) were used to inoculate solid modified complete media ( $1 \%$ glucose, $0.2 \%$ peptone, $0.1 \%$ yeast extract, $1 \%$ casamino acid, $0.6 \%$ $\mathrm{NaNO}_{3}, 0.05 \% \mathrm{KCl}, 0.05 \% \mathrm{MgSO}_{4}, 0.15 \% \mathrm{KH}_{2} \mathrm{PO}_{4}, 0.1 \%$ trace element [vol/vol], $0.1 \%$ vitamin supplement [vol/vol], $\mathrm{pH}$ 6.5 [all wt/vol, unless otherwise stated]). Plates were incubated at $25^{\circ} \mathrm{C}$ in the dark and radial growth area was measured at 7 days postinoculation. For hyphal-cell counting and celllength measurements, 7-day-old conidia were streaked on $10 \%$ $\mathrm{CM}$ agar slides and were incubated for $18 \mathrm{~h}$ at $25^{\circ} \mathrm{C}$. Hyphae grown from the conidia were stained with 100 ppm of Calcofluor white solution and were observed with a Zeiss Axio Imager A1 fluorescence microscope (Carl Zeiss). Hyphal cells grown from each conidium were counted and the lengths between septa were measured on ImageJ.

\section{Genotoxin and ROS sensitivity test.}

Primary inocula from solid MM, as mentioned above, were used to inoculate solid CM containing the following concentrations of genotoxins and ROS stressors: $0.1 \mu \mathrm{M} \mathrm{CPT}, 0.001 \%$ MMS, $10 \mathrm{mM} \mathrm{HU}, 2 \mathrm{mU}$ of bleomycin per milliliter, $5 \mathrm{mM}$ $\mathrm{H}_{2} \mathrm{O}_{2}$. Unsupplemented $\mathrm{CM}$ was used as negative control. Plates were incubated at $25^{\circ} \mathrm{C}$ in the dark and radial growth area was measured at 7 days postinoculation.

\section{Neutral comet assay.}

The neutral comet assay was carried out according to Olive and Banáth (2006), with some modifications. Freshly extracted protoplasts adjusted to $3 \times 10^{5}$ to $4 \times 10^{5}$ per milliliter in $20 \%$ sucrose were used immediately without freezing. As positive control for DNA DBSs, the wild-type mycelia were treated with $5 \mathrm{mU}$ of bleomycin per milliliter for $3 \mathrm{~h}$ during incubation with Trichoderma harzianum lysing enzyme (Sigma-Aldrich) in the protoplast extraction procedure. 'Comets' were stained with $0.25 \mu \mathrm{g}$ of ethidium bromide per milliliter and were viewed under Zeiss Axio Imager A1 fluorescence microscope (Carl Zeiss) with a filter set optimized for DsRed (20HE; excitation $546 \mathrm{~nm}$, emission $607 \mathrm{~nm}$ ). Comet images were analyzed with CometScore ( $n>100$ for the wild type, $\Delta$ Mortt109, and Rtt109c; $n=50$ for the bleomycin-positive control).

\section{Observations on asexual and pre-penetration development.}

Conidiation was measured using 7-day-old cultures on solid V8 medium. The entire colony was scraped in sterile distilled water and conidia were counted in a hemocytometer. Total number of conidia was calculated and divided by colony growth area to determine the number of conidia produced per square centimeter. Pre-penetration development was observed using conidial suspensions of $3 \times 10^{4}$ spores per millimeter dropped onto hydrophobic coverslips. Number of conidia in different stages of pre-penetration development was counted at 2, 4, 8, and 12 h later $(n \geq 100)$. Pre-penetration development were broadly divided into ungerminated conidium, germinated conidium, incipient appressorium, and mature, melanized appressorium.

\section{Pathogenicity assay.}

Conidia were harvested from an 8- to 10-day-old culture on oatmeal agar plate with sterilized distilled water. Conidial suspension was adjusted to $5 \times 10^{4}$ spores per milliliter in concentration and spray-inoculated on susceptible rice 
seedlings (cv. Nagdong) of three- to four-leaf stage. Inoculated plants were kept in a dew chamber for $24 \mathrm{~h}$ and were then moved to a growth chamber. Disease severity was assessed 7 days postinoculation. Wound-inoculation was carried out as follows. Rice leaf was wounded by pressing a pipette tip gently against the leaf surface and a drop of spore suspension was then placed right on top of the wound site. Lesion development was observed 5 to 7 days postinoculation. For sheath assay, rice leaf sheath was excised and was inoculated by conidia suspension $\left(1 \times 10^{4}\right.$ spores per milliliter $)$ on the adaxial surface. After 24 and $48 \mathrm{~h}$ of incubation in a moistened box, the sheath was trimmed to remove chlorophyll-enriched plant parts and the remaining epidermal layer of midvein (three- to four-cell layer thick) were used for observation under microscope.

\section{qRT-PCR and RNA sequencing.}

RNA extracted from 3-day-old mycelia grown in CM liquid was used for both qRT-PCR and RNA sequencing. qRT-PCR was performed using Rotor-Gene SYBR green PCR kit and Rotor-GeneQ (Qiagen). Paired-end sequencing of mRNAs was carried out on the Illumina platform. RNA-seq data generated and analyzed during the current study are available in National Center for Biotechnology Information GEO database (data was submitted and accession numbers will be given following assignment). TopHat was used to map the reads to the annotated $M$. oryzae transcripts, followed by analysis of transcript abundance and differential expression between the strains, using Cufflinks. Expression levels were calculated as fragments per kilobase per million reads. GO was carried out using topGO package in R programming (Alexa and Rahnenfuhrer 2016).

\section{ACKNOWLEDGMENTS}

We thank K. Min, B. Jeon, H. Chung, and A. Huh (Seoul National University) for advice and technical support.

\section{LITERATURE CITED}

Albaugh, B. N., Arnold, K. M., Lee, S., and Denu, J. M. 2011 Autoacetylation of the histone acetyltransferase Rtt109. J. Biol. Chem. 286:24694-24701.

Alexa A and Rahnenfuhrer J (2016). topGO: Enrichment analysis for gene ontology. R package version 2.30.0. BioConductor. Published online.

Belenky, P., Camacho, D., and Collins, J. J. 2013. Fungicidal drugs induce a common oxidative-damage cellular death pathway. Cell Reports 3:350-358.

Chen, C. C., Carson, J. J., Feser, J., Tamburini, B., Zabaronick, S., Linger, J., and Tyler, J. K. 2008. Acetylated lysine 56 on histone H3 drives chromatin assembly after repair and signals for the completion of repair. Cell 134:231-243.

Chi, M. H., Park, S. Y., Kim, S., and Lee, Y. H. 2009. A novel pathogenicity gene is required in the rice blast fungus to suppress the basal defenses of the host. PLoS Pathog. 5:e1000401.

Choi, J., Kim, K. T., Huh, A., Kwon, S., Hong, C., Asiegbu, F. O., Jeon, J., and Lee, Y. H. 2015. dbHiMo: A web-based epigenomics platform for histone-modifying enzymes. Database (Oxford) 2015:bav052.

Cools, H. J., Fraaije, B. A., Bean, T. P., Antoniw, J., and Lucas, J. A. 2007. Transcriptome profiling of the response of Mycosphaerella graminicola isolates to an azole fungicide using cDNA microarrays. Mol. Plant Pathol. 8:639-651.

Dahlin, J. L., Kottom, T., Han, J., Zhou, H., Walters, M. A., Zhang, Z., and Limper, A. H. 2014. Pneumocystis jirovecii Rtt109, a novel drug target for Pneumocystis pneumonia in immunosuppressed humans. Antimicrob. Agents Chemother. 58:3650-3659.

Dean, R., Van Kan, J. A., Pretorius, Z. A., Hammond-Kosack, K. E., Di Pietro, A., Spanu, P. D., Rudd, J. J., Dickman, M., Kahmann, R., Ellis, J., and Foster, G. D. 2012. The top 10 fungal pathogens in molecular plant pathology. Mol. Plant Pathol. 13:414-430.

Downs, J. A. 2008. Histone H3 K56 acetylation, chromatin assembly, and the DNA damage checkpoint. DNA Repair (Amst.) 7:2020-2024.

Driscoll, R., Hudson, A., and Jackson, S. P. 2007. Yeast Rtt109 promotes genome stability by acetylating histone H3 on lysine 56 . Science 315 : 649-652.
Fillingham, J., Recht, J., Silva, A. C., Suter, B., Emili, A., Stagljar, I., Krogan, N. J., Allis, C. D., Keogh, M. C., and Greenblatt, J. F. 2008. Chaperone control of the activity and specificity of the histone $\mathrm{H} 3$ acetyltransferase Rtt109. Mol. Cell. Biol. 28:4342-4353.

Guo, M., Chen, Y., Du, Y., Dong, Y., Guo, W., Zhai, S., Zhang, H., Dong, S., Zhang, Z., Wang, Y., Wang, P., and Zheng, X. 2011. The bZIP transcription factor MoAP1 mediates the oxidative stress response and is critical for pathogenicity of the rice blast fungus Magnaporthe oryzae. PLoS Pathog. 7:e1001302.

Guo, M., Guo, W., Chen, Y., Dong, S., Zhang, X., Zhang, H., Song, W., Wang, W., Wang, Q., Lv, R., Zhang, Z., Wang, Y., and Zheng, X. 2010. The basic leucine zipper transcription factor Moatf1 mediates oxidative stress responses and is necessary for full virulence of the rice blast fungus Magnaporthe oryzae. Mol. Plant-Microbe Interact 23:1053-1068.

Han, J., Zhou, H., Horazdovsky, B., Zhang, K., Xu, R. M., and Zhang, Z 2007. Rtt109 acetylates histone H3 lysine 56 and functions in DNA replication. Science 315:653-655.

Howard, R. J., and Valent, B. 1996. Breaking and entering: Host penetration by the fungal rice blast pathogen Magnaporthe grisea. Annu. Rev. Microbiol. 50:491-512.

Huang, K., Czymmek, K. J., Caplan, J. L., Sweigard, J. A., and Donofrio, N. M. 2011. HYR1-mediated detoxification of reactive oxygen species is required for full virulence in the rice blast fungus. PLoS Pathog. 7: e1001335.

Jessulat, M., Alamgir, M., Salsali, H., Greenblatt, J., Xu, J., and Golshani, A. 2008. Interacting proteins Rtt109 and Vps75 affect the efficiency of non-homologous end-joining in Saccharomyces cerevisiae. Arch. Biochem. Biophys. 469:157-164

Jiang, Y. W., and Kang, C. M. 2003. Induction of S. cerevisiae filamentous differentiation by slowed DNA synthesis involves Mec1, Rad53 and Swe1 checkpoint proteins. Mol. Biol. Cell 14:5116-5124.

Kankanala, P., Czymmek, K., and Valent, B. 2007. Roles for rice membrane dynamics and plasmodesmata during biotrophic invasion by the blast fungus. Plant Cell 19:706-724.

Keck, K. M., and Pemberton, L. F. 2011. Interaction with the histone chaperone Vps75 promotes nuclear localization and HAT activity of Rtt109 in vivo. Traffic 12:826-839.

Kottom, T. J., Han, J., Zhang, Z., and Limper, A. H. 2011. Pneumocystis carinii expresses an active Rtt109 histone acetyltransferase. Am. J. Respir. Cell Mol. Biol. 44:768-776.

Lercher, L., Danilenko, N., Kirkpatrick, J., and Carlomagno, T. 2018. Structural characterization of the Asf1-Rtt109 interaction and its role in histone acetylation. Nucleic Acids Res. 46:2279-2289.

Li, Q., Zhou, H., Wurtele, H., Davies, B., Horazdovsky, B., Verreault, A., and Zhang, Z. 2008. Acetylation of histone H3 lysine 56 regulates replication-coupled nucleosome assembly. Cell 134:244-255.

Lopes da Rosa, J., Bajaj, V., Spoonamore, J., and Kaufman, P. D. 2013. A small molecule inhibitor of fungal histone acetyltransferase Rtt109. Bioorg. Med. Chem. Lett. 23:2853-2859.

Lopes da Rosa, J., Boyartchuk, V. L., Zhu, L. J., and Kaufman, P. D. 2010 Histone acetyltransferase Rtt109 is required for Candida albicans pathogenesis. Proc. Natl. Acad. Sci. U.S.A. 107:1594-1599.

Masumoto, H., Hawke, D., Kobayashi, R., and Verreault, A. 2005. A role for cell-cycle-regulated histone $\mathrm{H} 3$ lysine 56 acetylation in the DNA damage response. Nature 436:294-298.

Muñoz-Galván, S., Jimeno, S., Rothstein, R., and Aguilera, A. 2013. Histone H3K56 acetylation, Rad52, and non-DNA repair factors control double-strand break repair choice with the sister chromatid. PLoS Genet 9:e1003237.

Ndindeng, S. A., Miki, S., Asano, K., and Sone, T. 2010. EGFP-Rhm51 foci enable the visualization and enumeration of DNA double-strand breaks in Magnaporthe oryzae. J. Gen. Plant Pathol. 76:377-381.

Neumann, H., Hancock, S. M., Buning, R., Routh, A., Chapman, L., Somers, J., Owen-Hughes, T., van Noort, J., Rhodes, D., and Chin, J. W. 2009. A method for genetically installing site-specific acetylation in recombinant histones defines the effects of H3 K56 acetylation. Mol. Cell 36:153-163.

O'Connell, M. J., Walworth, N. C., and Carr, A. M. 2000. The G2-phase DNA-damage checkpoint. Trends Cell Biol. 10:296-303.

Olive, P. L., and Banáth, J. P. 2006. The comet assay: A method to measure DNA damage in individual cells. Nat. Protoc. 1:23-29.

Ryder, L. S., and Talbot, N. J. 2015. Regulation of appressorium development in pathogenic fungi. Curr. Opin. Plant Biol. 26:8-13.

Salmon, T. B., Evert, B. A., Song, B., and Doetsch, P. W. 2004. Biological consequences of oxidative stress-induced DNA damage in Saccharomyces cerevisiae. Nucleic Acids Res. 32:3712-3723.

Saunders, D. G., Aves, S. J., and Talbot, N. J. 2010. Cell cycle-mediated regulation of plant infection by the rice blast fungus. Plant Cell 22:497-507. 
Scholes, D. T., Banerjee, M., Bowen, B., and Curcio, M. J. 2001. Multiple regulators of Ty1 transposition in Saccharomyces cerevisiae have conserved roles in genome maintenance. Genetics 159:1449-1465.

Shi, Q. M., Wang, Y. M., Zheng, X. D., Lee, R. T., and Wang, Y. 2007. Critical role of DNA checkpoints in mediating genotoxic-stress-induced filamentous growth in Candida albicans. Mol. Biol. Cell 18:815-826.

Skamnioti, P., and Gurr, S. J. 2009. Against the grain: Safeguarding rice from rice blast disease. Trends Biotechnol. 27:141-150.

Smerdon, M. J. 1991. DNA repair and the role of chromatin structure. Curr. Opin. Cell Biol. 3:422-428.

Stavropoulos, P., Nagy, V., Blobel, G., and Hoelz, A. 2008. Molecular basis for the autoregulation of the protein acetyl transferase Rtt109. Proc. Natl. Acad. Sci. U.S.A. 105:12236-12241.

Suwunnakorn, S., Cooper, C. R., Jr., Kummasook, A., Pongpom, M., Vanittanakom, P., and Vanittanakom, N. 2015. Role of the rttA gene in morphogenesis, stress response, and virulence in the human pathogenic fungus Penicillium marneffei. Med. Mycol. 53:119-131.

Talbot, N. J. 2003. On the trail of a cereal killer: Exploring the biology of Magnaporthe grisea. Annu. Rev. Microbiol. 57:177-202.

Tang, Y., Holbert, M. A., Wurtele, H., Meeth, K., Rocha, W., Gharib, M., Jiang, E., Thibault, P., Verreault, A., Cole, P. A., and Marmorstein, R. 2008. Fungal Rtt109 histone acetyltransferase is an unexpected structural homolog of metazoan p300/CBP. Nat. Struct. Mol. Biol. 15: 738-745.
Wilson, R. A., and Talbot, N. J. 2009. Under pressure: Investigating the biology of plant infection by Magnaporthe oryzae. Nat. Rev. Microbiol. 7:185-195. Wurtele, H., Tsao, S., Lépine, G., Mullick, A., Tremblay, J., Drogaris, P., Lee, E. H., Thibault, P., Verreault, A., and Raymond, M. 2010. Modulation of histone $\mathrm{H} 3$ lysine 56 acetylation as an antifungal therapeutic strategy. Nat. Med. 16:774-780.

Xhemalce, B., Miller, K. M., Driscoll, R., Masumoto, H., Jackson, S. P., Kouzarides, T., Verreault, A., and Arcangioli, B. 2007. Regulation of histone H3 lysine 56 acetylation in Schizosaccharomyces pombe. J. Biol. Chem. 282:15040-15047.

Yang, J., Yan, R., Roy, A., Xu, D., Poisson, J., and Zhang, Y. 2015. The ITASSER suite: Protein structure and function prediction. Nat. Methods 12:7-8.

Zhang, Z., Yang, Q., Sun, G., Chen, S., He, Q., Li, S., and Liu, Y. 2014 Histone H3K56 acetylation is required for quelling-induced small RNA production through its role in homologous recombination. J. Biol. Chem. 289:9365-9371.

\section{AUTHOR-RECOMMENDED INTERNET RESOURCES}

Center for Fungal Genetic Resources database: http://knrrb.knrrc.or.kr/ english/index.jsp?rrb=cfgr

I-TASSER server: https://zhanglab.ccmb.med.umich.edu/I-TASSER

Protein Data Bank: https://www.rcsb.org/pdb/home/home.do 